\title{
COMPUTATIONAL ANALYSIS OF A PROTOTYPE MARTIAN ROTORCRAFT EXPERIMENT
}

\author{
Kelly J. Corfeld* \\ Aerospace Engineering Department \\ The Pennsylvania State University \\ University Park, PA 16802 \\ kjc192@psu.edu \\ Roger C. Strawn ${ }^{\dagger}$ \\ Army/NASA Rotorcraft Division \\ Army Aeroflightdynamics Directorate (AMRDEC) \\ US Army Aviation and Missile Command \\ Ames Research Center, Moffett Field, CA \\ rstrawn@mail.arc.nasa.gov \\ Lyle N. Long ${ }^{\ddagger}$ \\ Aerospace Engineering Department \\ The Pennsylvania State University \\ University Park, PA 16802 \\ lnl@psu.edu
}

\begin{abstract}
This paper presents Reynolds-averaged Navier-Stokes calculations for a prototype Martian rotorcraft. The computations are intended for comparison with an ongoing Mars rotor hover test at NASA Ames Research Center. These computational simulations present a new and challenging problem, since rotors that operate on Mars will experience a unique low Reynolds number and high Mach number environment. Computed results for the 3-D rotor differ substantially from 2-D sectional computations in that the 3-D results exhibit a stall delay phenomenon caused by rotational forces along the blade span. Computational results have yet to be compared to experimental data, but computed performance predictions match the experimental design goals fairly well. In addition, the computed results provide a high level of detail in the rotor wake and blade surface aerodynamics. These details provide an important supplement to the expected experimental performance data.
\end{abstract}

\footnotetext{
* Graduate student, Member AIAA

$\dagger$ Research Scientist, Senior Member AIAA

$\ddagger$ Professor, Associate Fellow AIAA

This material is declared a work of the U.S.

Government and is not subject to copyright

protection in the United States.
}

\section{Introduction}

Recent NASA efforts have been devoted to the development of a vehicle for Mars exploration. A number of vehicle designs, including a Mars airplane and balloon designs, have been proposed for use in the exploration of Mars. Land vehicles, such as rovers, and space orbiters have already been studied. Recently, efforts have been directed towards the development of vertical lift vehicles, such as helicopters and tilt rotors, for use in Mars exploration.

Why use vertical lift vehicles for the exploration of Mars? They have the same advantages as those on Earth, and have vertical lift capabilities that are not available in other Mars exploration vehicles. The ability to hover, fly at low speeds, and take-off and land at unprepared sites gives vertical lift vehicles an advantage over other exploration methods such as the Mars airplane. Vertical lift vehicles have greater range and speed than a land rover, and will not be hampered by the rugged Martian terrain.

The Army/NASA Rotorcraft Division at NASA Ames Research Center has an ongoing effort to develop autonomous rotorcraft for the exploration of Mars. Preliminary studies of the design challenges associated with such an aircraft have been detailed in several papers $^{1-4}$. These studies have led to a NASA experiment that will test a prototype rotor in an environmental chamber that simulates the atmospheric conditions on Mars. Measurements of rotor thrust and torque will be made and micro-tuft surface flow visualizations on the rotor blades are also planned. 
In coordination with these experimental studies, the hover performance of this Mars rotor prototype has been computed using an overset-grid, Navier-Stokes CFD flow solver. Such simulations present new and challenging problems since rotors that operate on Mars will experience low Reynolds numbers and high Mach numbers. These conditions differ considerably from conventional rotor design points.

One of the objectives of this work is to examine whether traditional 2-D sectional aerodynamics methods can accurately model this Mars rotor problem. These sectional aerodynamics methods have been calibrated for conventional rotorcraft, where 2-D assumptions and rotor wake models typically work well prior to rotor stall. The Mars rotor has a unique geometry and will run in flow conditions that are outside of the validation ranges for 2-D lifting-line aerodynamics.

Recent 3-D hovering rotor CFD computations have shown good agreement with experimental performance results for conventional rotorcraft ${ }^{5-7}$. Unlike 2-D based aerodynamics methods, it is expected that the 3-D CFD method should also be able to accurately model the Mars rotor.

There is very little data on low Reynolds number aerodynamics and virtually none where the Mach number is high. Thus, many questions exist as to how these blades will perform in a Mars environment. The Navier-Stokes calculations will complement the experimental data, providing basic information and validated analysis tools for future Mars rotor designs.

The Navier-Stokes solver being used for this analysis is OVERFLOW-D ${ }^{8}$, which was developed by the Army/NASA Rotorcraft Division under Department of Defense funding. The OVERGRID package ${ }^{8}$ was used to develop a series of overset structured grids for the Navier-Stokes CFD analysis. A more detailed description of all of this work can be found in Reference 9.

\section{Experimental setup}

The prototype Mars rotor is currently being tested at NASA Ames Research Center. The test is being conducted in a large environmental chamber, which has the ability to closely simulate the Mars surface atmospheric conditions. A comparison of Earth and Mars atmospheric properties is given in Table $1^{10}$. The chamber has the ability to match the Mars mean surface atmospheric pressure, which allows the experiment to match the Mars operating Reynolds number and Mach number.
Table 1. Comparison of Mean Atmospheric Properties

\begin{tabular}{|l|c|c|}
\hline & MARS & EARTH \\
\hline Density $\left(\mathrm{kg} / \mathrm{m}^{3}\right)$ & 0.00155 & 1.23 \\
\hline Pressure $(\mathrm{Pa})$ & 636 & 101,300 \\
\hline Temperature $(\mathrm{K})$ & 214 & 288 \\
\hline Speed of Sound $(\mathrm{m} / \mathrm{s})$ & 230 & 340 \\
\hline Atmospheric Gases & $95 \% \mathrm{CO}_{2}$ & $78 \% \mathrm{~N}_{2}$ \\
& $2.7 \% \mathrm{~N}_{2}$ & $21 \% \mathrm{O}_{2}$ \\
\hline Gamma, $\gamma$ & 1.3 & 1.4 \\
\hline
\end{tabular}

This proof-of-concept rotor was designed to meet the challenges of operating in the Martian environment. Rotorcraft operating on Mars will encounter a unique combination of low Reynolds number and compressible flow aerodynamics. The experimental test conditions have a tip Mach number of 0.65 and a Reynolds number of 50,000, based on tip speed and blade chord. A fairly conventional low Reynolds number airfoil section (Eppler $387^{11}$ ) was chosen for the experimental rotor.

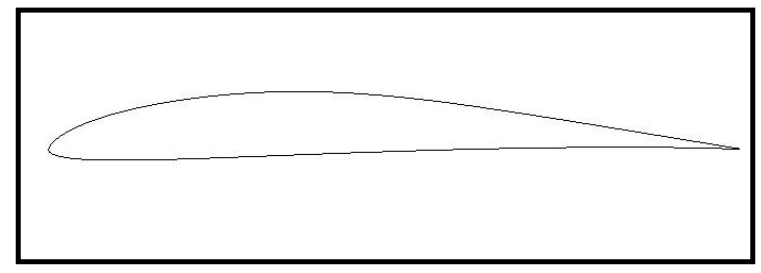

Figure 1. Eppler 387 airfoil

Figure 2 shows the Mars rotor hover test stand. Notable features include the low aspect ratio blades and the $40 \%$ root cut out to accommodate blade folding and telescoping in transport and deployment. Also, the experimental and computational studies model an isolated rotor only, while the actual design will consist of two coaxial rotors. Table $2^{12}$ gives the complete list of the Mars rotor specifications. 


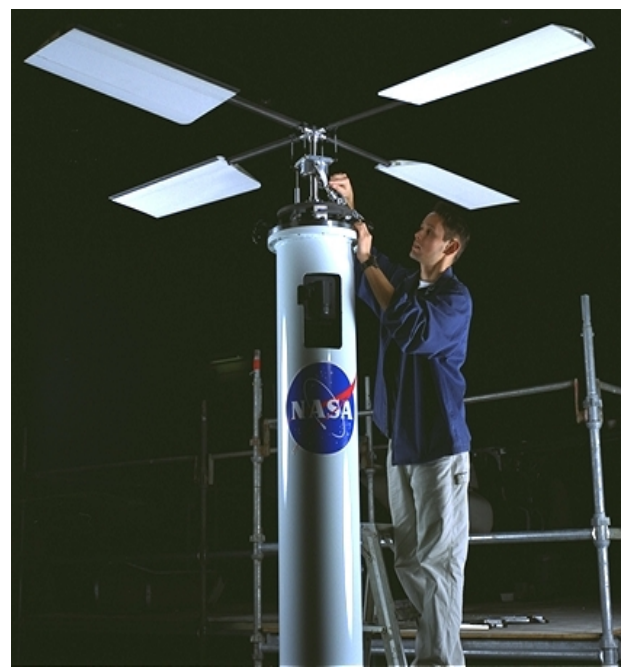

Figure 2. Mars Rotor Hover Test

\section{Computational Methods}

OVERFLOW-D is a scalable Reynolds-averaged Navier-Stokes solver that uses overset structured grids to accommodate complex geometries. OVERFLOW-D is based on OVERFLOW ${ }^{13}$, but has been enhanced to allow for moving body applications, facilitate accuracy control via solution adaption, and run efficiently on scalable parallel computers. Modifications were also made to OVERFLOW-D to facilitate the solution of hovering rotor problems. These modifications include the implementation of periodicity and the addition of two-cell grid overlaps for increased spatial accuracy ${ }^{5}$. The Mars rotor calculations for this paper were computed using a Lower Upper-Symmetric GaussSeidel (LUSGS) scheme with fourth-order centraldifferencing and local time stepping.

OVERFLOW-D is used in conjunction with the OVERGRID, a software package for overset gridgeneration and solution analysis. The package includes the grid generation tool called OVERGRID, which is a graphical user interface used to generate grid systems. OVERGRID performs numerous operations such as checking and processing input geometry, generating surface and volume grids, analyzing grid quality using various diagnostics, and creating input parameter files for the domain connectivity program and the flow solver.
Table 2. Mars Rotor Description

\begin{tabular}{|c|c|}
\hline Number of Blades & 4 \\
\hline Rotor Diameter & $2.438 \mathrm{~m}$ \\
\hline $\begin{array}{l}\text { Blade Root Cut-Out } \\
\text { (To simulate blade } \\
\text { telescoping required } \\
\text { for storage/transport) }\end{array}$ & $40 \%$ blade span \\
\hline $\begin{array}{l}\text { Disk Loading } \\
\text { (Nominal '1G') }\end{array}$ & $4 \mathrm{~N} / \mathrm{m}^{2}$ \\
\hline Tip Mach Number & 0.65 \\
\hline $\begin{array}{l}\text { Blade Tip Reynolds } \\
\text { Number }\end{array}$ & 50,000 \\
\hline $\begin{array}{l}\text { Thrust Coefficient, } \\
\text { CT (Nominal '1G') }\end{array}$ & 0.0108 \\
\hline $\begin{array}{l}\text { Mean Blade Lift } \\
\text { Coefficient }\end{array}$ & 0.4 \\
\hline Blade Chord & $\begin{array}{l}0.3048 \mathrm{~m} \text { (constant) from } \\
40 \% \text { radial station outward }\end{array}$ \\
\hline Rotor Solidity & 0.191 \\
\hline $\begin{array}{l}\text { Blade Linear Twist } \\
\text { Rate }\end{array}$ & $\begin{array}{l}0 \text { deg. out to } 40 \% \text { span; } \\
+2.4 \text { to }-2.4 \text { deg. from } 40 \text { to } \\
100 \% \text { span. }\end{array}$ \\
\hline Blade Weight & $0.35 \mathrm{~kg}$ per blade \\
\hline $\begin{array}{l}\text { First Fundamental } \\
\text { Elastic Modes }\end{array}$ & $\begin{array}{l}1.264 \text { per rev - first flap } \\
\text { mode; } \\
1.118 \text { per rev - first lag } \\
\text { mode; } \\
2.310 \text { per rev - first torsion }\end{array}$ \\
\hline $\begin{array}{l}\text { Outer Blade Span } \\
\text { Airfoil Section }\end{array}$ & Eppler 387 \\
\hline Spar Section & $\begin{array}{l}\text { Circular tube with } \\
\text { chordwise flat plate stiffener } \\
\text { (30\% chord) }\end{array}$ \\
\hline Blade Construction & $\begin{array}{l}\text { Airfoil fairing is milled } \\
\text { foam with internal cavities; } \\
\text { Circular graphite tube spar } \\
\text { across complete span of } \\
\text { blade; } \\
45 \text { deg. graphite chordwise } \\
\text { flat plate stiffeners from 5\% } \\
\text { to } 40 \% \text { station; } \\
\text { fiberglass leading edge cap } \\
\text { on outer blade section } \\
\text { airfoil fairing }\end{array}$ \\
\hline $\begin{array}{l}\text { Rotor Hub } \\
\text { Configuration }\end{array}$ & $\begin{array}{l}\text { Rigid/cantilevered hub, with } \\
\text { tension/torsion straps, dry } \\
\text { contact pitch bearings, and } \\
\text { pitch arms at } 5 \% \text { radial } \\
\text { station }\end{array}$ \\
\hline
\end{tabular}




\section{Mars Rotor Grid System}

OVERFLOW-D discretizes the problem domain into near-body and off-body grids ${ }^{14}$. The near-body grids consist of curvilinear body-fitted meshes to capture viscous effects over surfaces. The off-body grids consist of uniform Cartesian meshes with varying levels of resolution. These Cartesian grids allow for high accuracy near the rotor surfaces while maintaining computational efficiency in the far field. The flow solver interpolates boundary data between adjacent overset meshes.

The near-body grid for these calculations consists of individual body-fitted curvilinear grids for the hub, spar, and rotor blade, as shown in Fig. 3. The near-body grids for the single rotor blade contain 1,888,282 grid points.

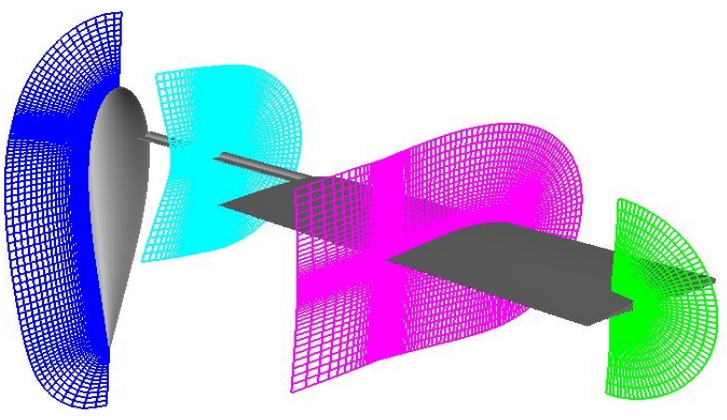

Figure 3. Near-body grid system

The off-body grid system in OVERFLOW-D uses uniform Cartesian grids that surround the near-body grids with varying levels of resolution. The grids become coarser as the outer computational boundary is approached. The off-body grid system for the Mars rotor is shown in Figure 4. The off-body grids contain $6,376,292$ points that are distributed in four levels of spacing, where the grid spacing doubles with each successive level. Figure 4 shows the four off-body grid levels. The " $\Delta s$ " represents the grid spacing within level one, and the corresponding grid spacing for each successive level is indicated in the figure. This scheme allows the outer grid boundaries to be located far away from the rotor with an efficient distribution of grid points.

Periodicity has been implemented in OVERFLOW-D and this allows a four-bladed rotor system to be modeled with a single rotor blade. Thus, the size of the computational domain can be reduced by a factor of four. The total calculation consists of 21 total grids and 8,264,574 total grid points.

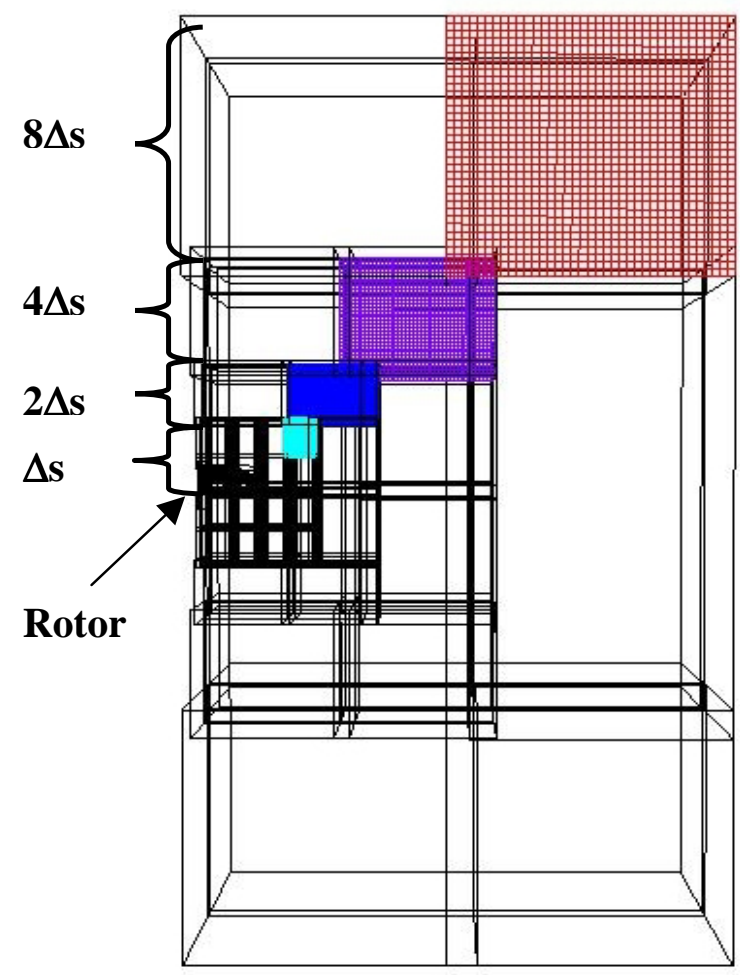

Figure 4. Off-body grid system

\section{Computational Results}

\section{2-Dimensional Calculations}

Prior to carrying out the 3-D rotor calculations, a 2D Navier-Stokes CFD analysis of the Eppler 387 airfoil was completed. These 2-D calculations were done using ARC2D, a 2-D Navier-Stokes flow solver ${ }^{15}$. The computational grid used for these calculations is shown in Figure 5. Fine viscous spacing was used at the wall with the first grid point located at $\mathrm{y}^{+}<1$ for all of the 2 D and 3-D computations.

ARC2D calculations were initially run at the experimental conditions of Mach number 0.5 (corresponding to the three-quarters span location of the rotor blade) and Reynolds number 50,000, for a series of angles of attack. Cases were run fully laminar, transitioned and fully turbulent. The transitional cases were simulated by specifying a turbulent region for all chordwise locations beyond a fixed transition point (typically $25 \%$ of chord). 


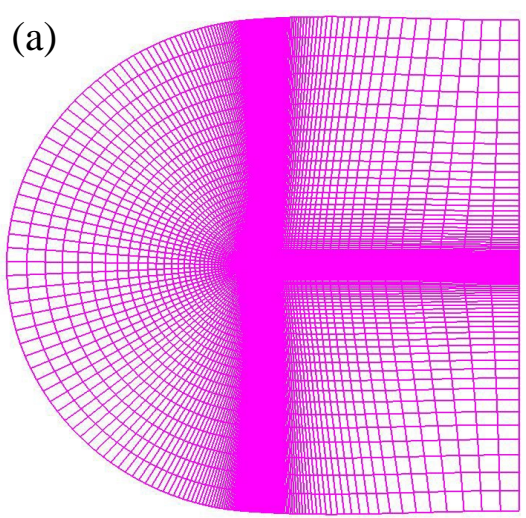

(b)

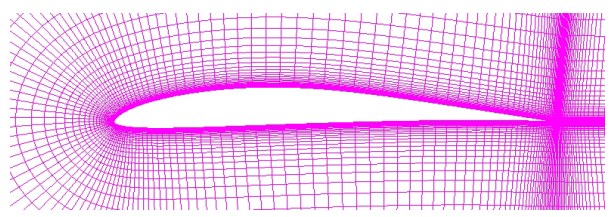

Figure 5. (a) 2-D grid on Eppler 387 airfoil (b) close up of grid on Eppler 387 airfoil

Unsteady separated flow was seen for all cases (laminar, transitioned and turbulent), and at all angles of attack. Figure 6 shows the density contours for the laminar flow case at zero degrees angle of attack. Note that unsteady vortices are being shed off the trailing edge of the airfoil.

This unsteadiness is consistent with recent observations by Mayda et al. ${ }^{16}$ in a wind turbine airfoil study. They found that unsteady calculations on the Eppler 387 airfoil resulted in high frequency unsteadiness. When time averaged, the results agreed well with experimental data. They also found that this trailing edge unsteadiness did not cause a large drag penalty. The transitioned and turbulent cases exhibited a separated region near the trailing edge of the airfoil. However, the large eddies that were seen in the laminar case were not present.

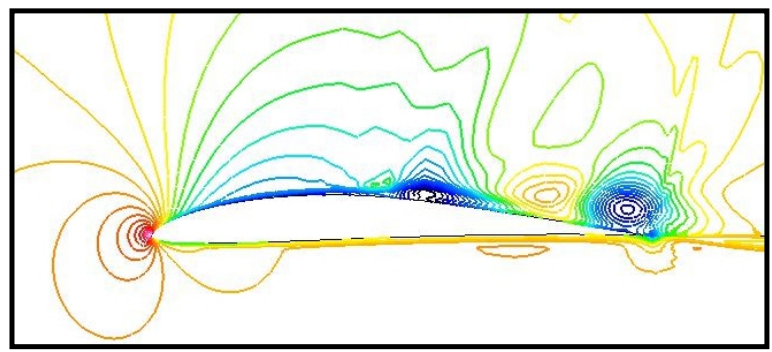

Figure 6. Eddies shed off trailing edge of Eppler 387 in laminar calculation (density contours)
In addition, ARC2D calculations were also completed for comparison with low Reynolds number experimental data obtained for the Eppler 387 airfoil in the NASA Langley Low-Turbulence Pressure Tunnel $(\mathrm{LTPT})^{11}$. Figure 7 compares the calculations and experimental data for a series of Reynolds numbers.

In Fig. 7, the ARC2D calculations match the experimental data more closely as the Reynolds number increases. The cause of this behavior stems from the transitional flow and separation bubbles that occur at these low Reynolds numbers. It was not expected that the ARC2D calculations would be able to match the data at transitional Reynolds numbers as this type of flow is typically very difficult to predict. Unfortunately, the Martian rotorcraft will operate at or near these transitional Reynolds numbers.

\section{3-Dimensional Calculations}

Hovering rotor OVERFLOW-D solutions were computed for a series of collective pitch angles. Cases were run as fully laminar, transitioned, and fully turbulent, at a hover tip Mach number of 0.65 and a tip Reynolds number of 50,000. As in the ARC2D calculations, boundary layer transition was modeled by specifying turbulent flow in the region aft of a prescribed transition point.

Results for the transition case use an 8.4 degrees collective pitch. This 8.4 degres angle is the estimated collective pitch required to produce a thrust coefficient greater than 0.1 and a hover figure of merit greater than 0.4 . For rotors, the figure of merit represents the ratio of ideal to actual power required for hover

The $8.3 \mathrm{M}$ grid point computations were run on 32 processors of an SGI Origin 2000 parallel computer. Overall thrust on the rotor was used as the measure of convergence to steady-state for the computation. All cases, laminar, transitional and turbulent, converged to approximately the same steady-state thrust coefficient. This steady-state behavior contrasts with the unsteadiness in the 2-dimensional analysis. Figure 8 shows a sample convergence plot for a typical case. The calculations were run for approximately 30,000 iterations, though the thrust showed very little variation after 15,000 iterations. A single calculation for 30,000 iterations required approximately 48 hours on 32 SGI Origin processors (1536 CPU hours). 

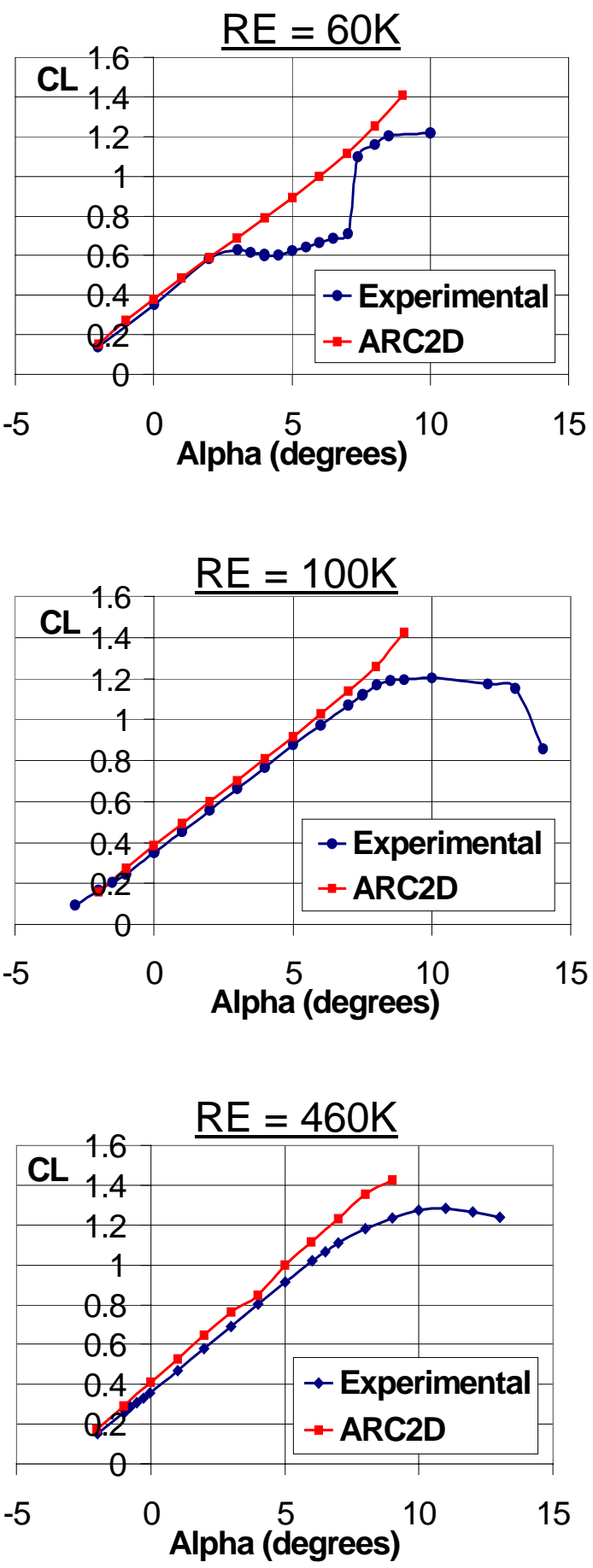

Figure 7. Lift coefficient comparison of ARC2D calculations and Langley LTPT data

Figure 9 shows a comparison of the pressure coefficients from the 3-D rotor computations and the Langley LTPT Eppler 387 airfoil (2-D) experiment. Both plots correspond to a lift coefficient of 0.28 . It can be seen that the upper surface pressure coefficients show poor agreement. This is due to the existence of a laminar separation bubble on the airfoil, as indicated by the flat region on the plot of the experimental pressure coefficient. The CFD code used in the 3-D rotor computations is unable to predict laminar separation bubbles, so it was not expected that the 3-D calculations would match the 2-D data. CFD codes, at present, are not able to predict low Reynolds number aerodynamics very well, as we showed with the ARC2D solutions. An examination of the flow near the surface of the blade, however, shows that the behavior of the flow may be altered due to rotational and 3-D effects. This effectively produces a flowfield that appears to be similar to higher Reynolds number results. Therefore, the calculations may be more accurate than one would expect. In addition, one must be careful in inferring too much about rotor performance from 2-D airfoil calculations or experiments at these low Reynolds numbers.

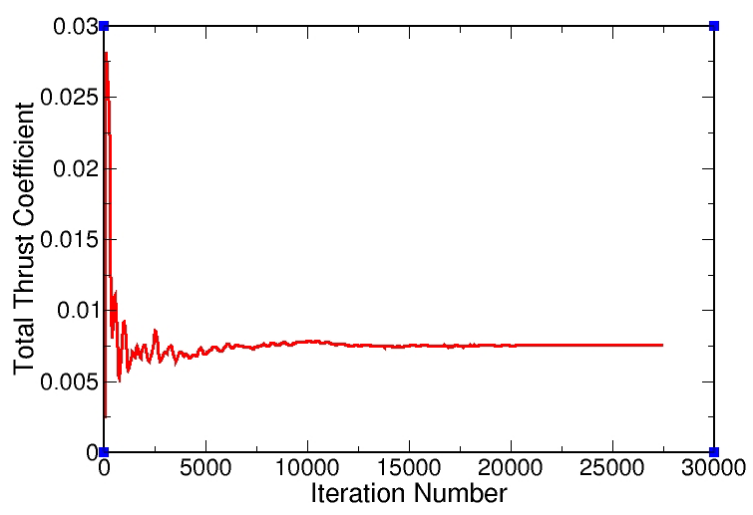

Figure 8. Typical thrust convergence

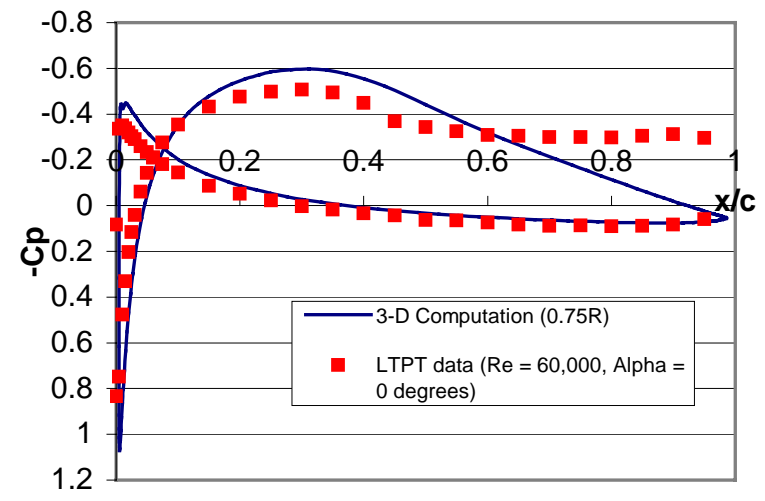

Figure 9. Comparison of pressure coefficient for 3-D computation and LTPT experiment 
Examination of the flow field near the blade reveals some interesting flowfield features. Figure 10 shows the trailing edge flow details at the three-quarter span location of the rotor blade for both the laminar and turbulent cases. Both cases exhibit a steady thin region of separated flow, which is slightly thicker in the laminar calculation.

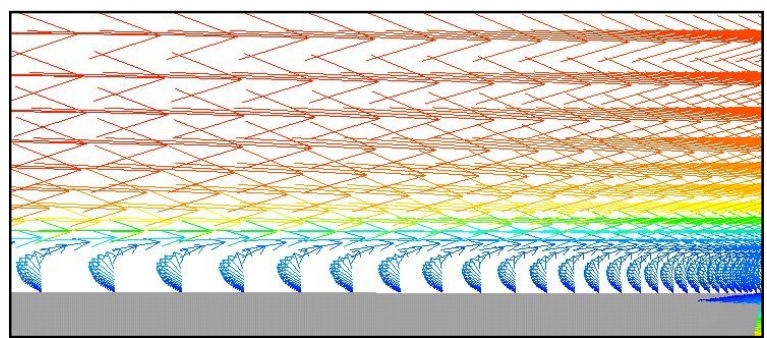

(a)

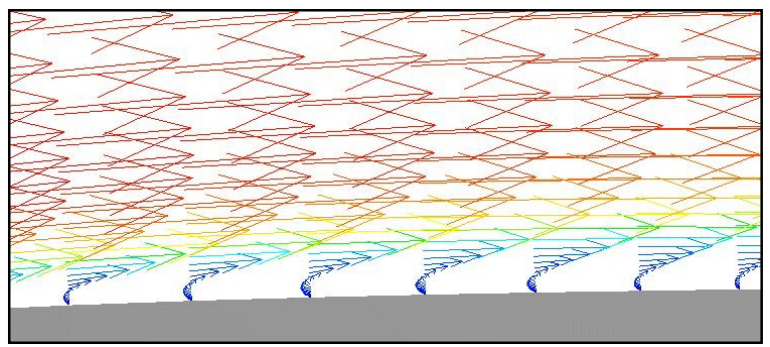

(b)

Figure 10. Velocity vectors near the trailing edge for (a) laminar and (b) turbulent calculations

Compared to the 2-D calculations, the 3-D boundary layer is thinner and steadier. Figure 11 shows the flow on the surface of the blade for both the laminar and turbulent cases. In both cases, rotational and 3-D effects cause a radial outflow near the blade surface in the separated regions. This radial outflow extends from the blade root to the blade tip, where it is absorbed by the tip vortex.

The thinning of the boundary layer and radial outflow are consistent with the stall delay phenomenon that is often seen in propellers and rotors. Harris ${ }^{18}$ observed this phenomenon in the 1960's in a general study of radial flow effects on rotating blades. Since then, this phenomenon has been seen, both experimentally and computationally, in studies involving tilt-rotor blades ${ }^{18}$, wind turbine blades ${ }^{19}$, and highly twisted rotors ${ }^{20}$. However, due to the low Reynolds number, the phenomenon seen for the Mars rotor is more pronounced and more extensive than that seen for the rotors used in these past studies.

In each study where this discrepancy between 2-D and 3-D predictions occurs, there are two main observations: (1) rotational effects allow the flow on the surface of the blade to remain attached longer and (2) radial flow due to rotation thins the separated boundary layer.

The first observation is referred to as stall delay, and this phenomenon is modeled in many comprehensive rotor analysis codes. In these models, the stall is delayed even when the 2-D airfoil data indicate stall.

The second observation describes more of a stall modification than stall delay, and this is the dominant phenomenon for the 3-D Mars rotor calculations. The rotational and 3-D effects on the rotor cause a thinning of the separated boundary layer, which stabilizes the overall flowfield and accounts for the differences between the unsteady 2-D and steady 3-D computed results.

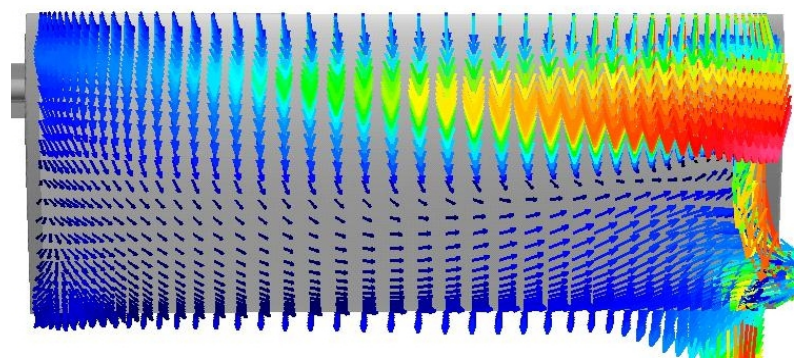

(a)

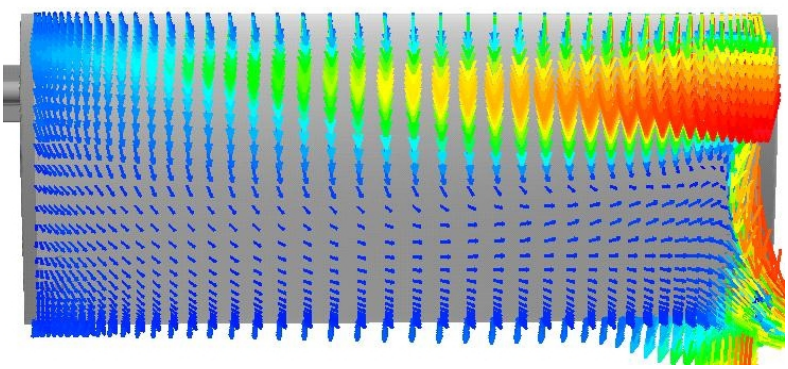

(b)

Figure 11. Velocity vectors on the blade surface for (a) laminar and (b) turbulent calculations

Figure 12 shows performance predictions for the Mars rotor in hover for a series of collective pitch angles. All of the cases, fully laminar, transitioned and fully turbulent, produced approximately the same performance results. Note that, while a series of laminar and turbulent cases were completed, only one transitioned case was run. It seemed unnecessary to run the transitioned case for the various collective pitch angles due to the similarity in the performance results for the fully laminar and fully turbulent cases.

The design points shown in Figure 12 were computed using blade element momentum methods. Note that the computed thrust coefficients and figures of merit are slightly higher than the design point. 

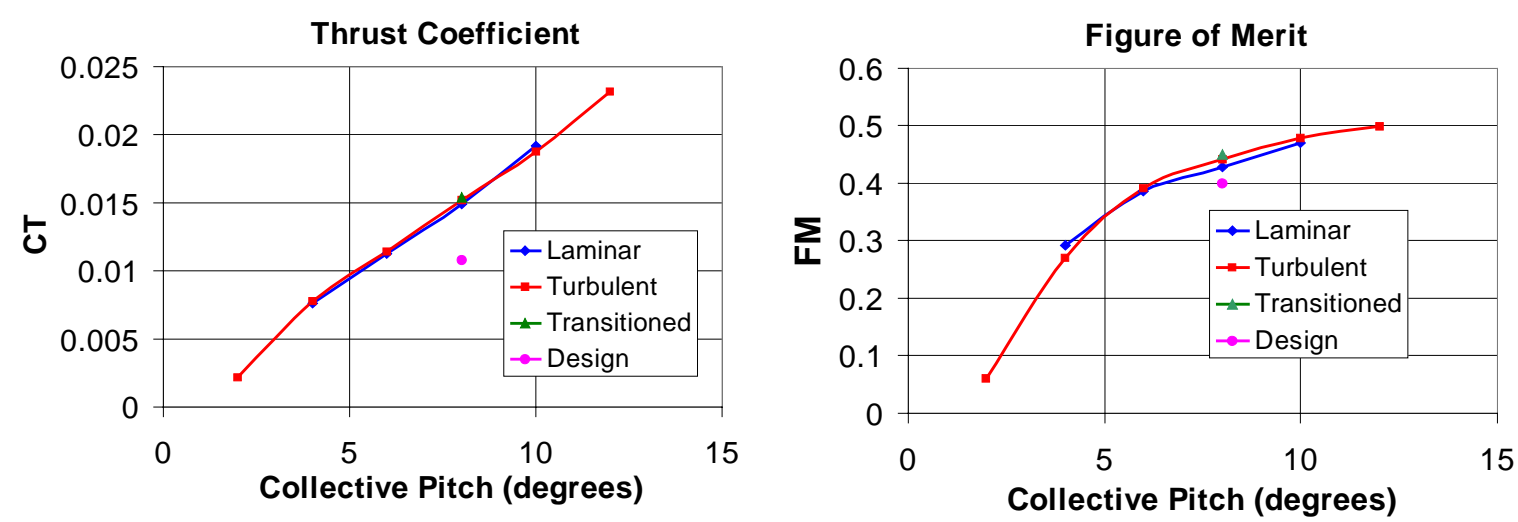

Figure 12. Performance predictions for Mars rotor in hover $\left(M_{\text {tip }}=\mathbf{0 . 6 5}\right.$, $\left.\operatorname{Re}_{\text {tip }}=50,000\right)$. (a) Thrust Coefficient (b) Figure of Merit

Figure 13 shows a section of the computed vortex wake system for the Mars rotor. This picture shows that the computations have captured a number of individual tip vortices, as well as their corresponding vortex sheets. Also, this vortex wake system exhibits unique characteristics that are not typically seen in rotor wake systems. Due to the $40 \%$ root cut out, a strong root vortex is present. Also, there is minimal wake contraction, which probably results from the low disc loading and the presence of the root vortices.

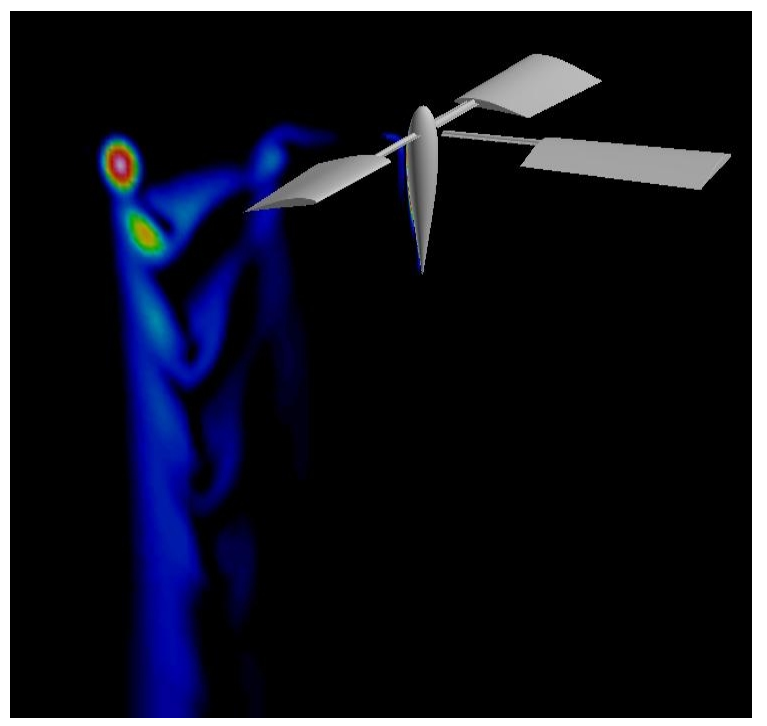

Figure 13. Computed vorticity magnitude contours on a cutting plane located $60^{\circ}$ behind the rotor blade

\section{$\underline{\text { Summary and Conclusions }}$}

This paper presents Reynolds-averaged NavierStokes calculations for a prototype Martian rotorcraft. The first step in this analysis was the creation of an overset grid system. This grid was used in the present study, and may be used again for future computational studies of this rotor system.

Solutions for hovering rotor performance at Mars experimental flow conditions were produced for a series of collective pitch angles. These simulations present results for a unique operating environment for rotorcraft. Rotors that operate on Mars will experience low Reynolds number and high Mach number aerodynamics, a combination not typically seen in rotorcraft. This is an area that has not yet been investigated until recently, and the CFD results provide insight into the physics of these flows. In addition, the CFD results have already proven useful as an aid to the experimentalists in their pre-test planning.

Computational results for the Mars rotor show significant three-dimensional effects. Most likely, these 3-D effects can't be modeled in a predictive way with 2-D sectional aerodynamics methods. Examples of these 3-D effects are the stall modifications due to rotation, and the presence of strong root vortices at the blade cutout. These root vortices contribute significantly to the inflow from the rotor wake and tend to diminish the wake contraction.

In the near future, the computational results will be compared to the experimental data, and these comparisons will provide an assessment and validation of these CFD tools for use in future Mars rotor designs. 
The rotor that was designed for the hover test was simply a proof-of-concept design. Future computational efforts will test possible design changes in order to improve performance. In addition, the CFD calculations will also be useful to help extrapolate the experimental data to the true Martian atmosphere.

\section{$\underline{\text { References }}$}

1. Young, L.A., Aiken, E.W., Gulick, V., Manicinelli, R., and Briggs, G.A., "Rotorcraft as Mars Scouts," 2002 IEEE Aerospace Conference, Big Sky, MT, March 2002.

2. Young, L.A. and Aiken, E.W., "Vertical Lift Planetary Aerial Vehicles: Three Planetary Bodies and Four Conceptual Design Cases," European Rotorcraft Forum, September, 2001.

3. Young, L.A., "Vertical Lift - Not Just for Terrestrial Flight," AHS/AIAA/RaeS/SAE International Powered Lift Conference, Arlington, VA, October 2000.

4. Young, L.A., Chen, L.A., Aiken, E.W., and Briggs, G.A., "Design Opportunities and Challenges in the Development of Vertical Lift Planetary Aerial Vehicles," American Helicopter Society Vertical Lift Aircraft Design Conference, San Francisco, CA, January 2000.

5. Strawn, R.C. and Djomehri, M.J., "Computational Modeling of Hovering Rotor and Wake Aerodynamics," American Helicopter Society (AHS) $57^{\text {th }}$ Annual Forum, Washington, DC, May 2001.

6. Kunz, P.J. and Strawn, R.C., "Analysis and Design of Rotors at Ultra-low Reynolds Numbers," $40^{\text {th }}$ AIAA Aerospace Sciences Meeting, Reno, NV, Jan. 11-14, 2002.

7. Potsdam, M.A. and Strawn, R.C., "CFD Simulations of Tiltrotor Configurations in Hover," American Helicopter Society $58^{\text {th }}$ Annual Forum, Montreal, Canada, June 2002.

8. Chan, W., Meakin, R., and Potsdam, M., "CHSSI Software for Geometrically Complex Unsteady Aerodynamics Applications," AIAA 2001-0539, AIAA $39^{\text {th }}$ Aerospace Sciences, Meeting, Reno, NV, Jan. 2001.

9. Corfeld, K.J., "Computational Analysis of a Prototype Martian Rotorcraft Experiment," M.S.
Thesis, Pennsylvania State University, Department of Aerospace Engineering, May 2002.

10. Lodders, K. and Fegley, Jr., B., The Planetary Scientist's Companion, Oxford University Press, 1998.

11. McGhee, R.J., et al, "Experimental Results for the Eppler 387 Airfoil at Low Reynolds Number in the Langley Low-Turbulence Pressure Tunnel," NASA TM-4062, 1988.

12. Young, L.A., Aiken, E.W., Derby, M.R., Demblewski, R., and Navarette, J., "Experimental Investigation and Demonstration of Rotary-Wing Technologies for Flight in the Atmosphere of Mars," American Helicopter Society $58^{\text {th }}$ Annual Forum, Montreal, Canada, June 2002.

13. Buning, P.G., Jespersen, D.C., Pulliam, T.H., Chan, W.M., Slotnick, J.P., Krist, S.E., and Renze, K.J., "OVERFLOW User's Manual, Version 1.8g," NASA Langley Research Center, March 1999.

14. Meakin, R., "Automatic Off-Body Grid Generation for Domains of Arbitrary Size," AIAA 2001-2536, $15^{\text {th }}$ AIAA CFD Conference, Anaheim, CA, June 2001.

15. Pulliam, T., ARC2D V3.00, NASA Ames Research Center, Copyright 1992.

16. Mayda, E.A. et al, "Bubble Induced Unsteadiness on Wind Turbine Airfoils," AIAA Wind Energy Conference, Reno, NV, 2002.

17. Harris, F.D., "Preliminary Study of Radial Flow Effects on Rotating Blades," Journal of the American Helicopter Society, Volume 11, No. 3, 1966.

18. Narramore, J.C. and Vermeland, R., "Use of Navier-Stokes Code to Predict Flow Phenomena Near Stall as Measured on a 0.658-Scale V-22 Tiltrotor Blade," AIAA 20 ${ }^{\text {th }}$ Fluid Dynamics, Plasma Dynamics and Lasers Conference, Buffalo, NY, June 1989.

19. Snel, H. and van Holten, Th., "Review of Recent Aerodynamics Research on Wind Turbines with Relevance to Rotorcraft," AGARD FDP Symposium on Aerodynamics and Aeroacoustics of Rotorcraft, Berlin, Germany, 1994. 
20. Yamauchi, G.K. and Johnson, W.R., "NavierStokes Calculations for a Highly-Twisted Rotor Near Stall," American Helicopter Society Aeromechanics Specialists Conference, San Francisco, CA, January 1994. 